\title{
Large SDSS Quasar Groups and Their Statistical Significance
}

\author{
Changbom Park $^{1}$, Hyunmi Song ${ }^{1}$, Maret Einasto $^{2}$, Heidi Lietzen ${ }^{3,4}$, and Pekka Heinämäki ${ }^{5}$ \\ ${ }^{1}$ School of Physics, Korea Institute for Advanced Study, Heogiro 85, Seoul 130-722, Korea \\ cbp@kias.re.kr, hmsong@kias.re.kr \\ ${ }^{2}$ Tartu Observatory, 61602 Toravere, Estonia; maret@to.ee \\ ${ }^{3}$ Instituto de Astrofísica de Canarias, E-38205 La Laguna, Tenerife, Spain; hlietzen@iac.es \\ ${ }^{4}$ Universidad de La Laguna, Dept. Astrofísica, E-38206 La Laguna, Tenerife, Spain \\ ${ }^{5}$ Tuorla Observatory, University of Turku, Väisäläntie 20, Piikkiö, Finland; pekheina@utu.fi
}

Received Jauuary 31, 2015; accepted February 5, 2015

\begin{abstract}
We use a volume-limited sample of quasars in the Sloan Digital Sky Survey (SDSS) DR7 quasar catalog to identify quasar groups and address their statistical significance. This quasar sample has a uniform selection function on the sky and nearly a maximum possible contiguous volume that can be drawn from the DR7 catalog. Quasar groups are identified by using the Friend-of-Friend algorithm with a set of fixed comoving linking lengths. We find that the richness distribution of the richest 100 quasar groups or the size distribution of the largest 100 groups are statistically equivalent with those of randomly-distributed points with the same number density and sky coverage when groups are identified with the linking length of $70 \mathrm{~h}^{-1} \mathrm{Mpc}$. It is shown that the large-scale structures like the huge Large Quasar Group (U1.27) reported by Clowes et al. (2013) can be found with high probability even if quasars have no physical clustering, and does not challenge the initially homogeneous cosmological models. Our results are statistically more reliable than those of Nadathur (2013), where the test was made only for the largest quasar group. It is shown that the linking length should be smaller than $50 h^{-1} \mathrm{Mpc}$ in order for the quasar groups identified in the DR7 catalog not to be dominated by associations of quasars grouped by chance. We present 20 richest quasar groups identified with the linking length of $70 h^{-1} \mathrm{Mpc}$ for further analyses.
\end{abstract}

Key words: large-scale structure of universe - cosmology: observations — quasars: general

\section{INTRODUCTION}

The size and mass of large-scale structures ${ }^{1}$ (hereafter LSS) in the universe have been often used as measures of amplitude of density fluctuations on large scales. The CfA Great Wall discovered by de Lapparent et al. (1986) in the CfA2 survey and the Sloan Great Wall in the SDSS survey (Gott et al. 2005) cast doubt on the SCDM model (Geller \& Huchra 1989) and the $\Lambda$ CDM model (Sheth \& Diaferio 2011), respectively. It has been argued that the universe did not have enough time for such large structures to form by the present epoch in these cosmological models, and that the gravitational instability theory for structure formation or the Cosmological Principle adopting large-scale homogeneity may not be valid (see also Clowes et al. 2012; Horvath et al. 2013).

On the other hand, a strong support for the Cosmological Principle comes from the smooth cos-

\footnotetext{
Corresponding AUthor: H. Song

${ }^{1}$ We use 'large-scale structure' as a generic term for structures larger than galaxy clusters. In this terminology the large-scale structures of the universe are classified into high-density and low-density large-scale structures. The high-density large-scale structures are classified into categories of superclusters, filaments, and walls depending on their internal density and morphology. The low-density large-scale structures are voids, and classified into bubbles, and tunnels depending on their morphology. In this scheme all the names that have been used to describe 'large' structures in the universe are unified.
}

mic microwave background radiation, which shows anisotropies that agrees astonishingly well with those of the initially homogeneous isotropic $\Lambda \mathrm{CDM}$ model (Clifton et al. 2012; Planck collaboration XV 2014). The fluctuation of the galaxy number density on large scales is also found to agree with the prediction of the $\Lambda$ CDM model (Scrimgeour et al. 2012).

It is expected that LSS of the universe contain a wealth of information on primordial density fluctuations and the history of their growth. This is because they represent density fluctuations on linear scales. It is then very important to rigorously study the physical properties and cosmological meaning of observed superstructures of the universe (Park 1990; Park et al. 2012; Einasto et al. 2011, 2014).

Recently, Clowes et al. (2013) reported a finding of a very large quasar group in the SDSS DR7 quasar catalog (Schneider et al. 2010) with characteristic size of $\sim 350 h^{-1} \mathrm{Mpc}$, longest dimension of $\sim 870 h^{-1} \mathrm{Mpc}$, membership of 73 quasars, and mean redshift $\bar{z}=1.27$. They claimed that the quasar group, named U1.27, is the largest 'structure' currently known in the universe. They argued that their finding might raise a question on validity of the large-scale homogeneity assumption, which is, in the form of Cosmological Principle, one of the fundamental assumptions of the standard cosmology. Their claim falls on the same kind of view applied to CfA and Sloan Great Walls. 
However, Nadathur (2013) pointed out that the statistical significance of the huge quasar group has not been properly studied in Clowes et al. (2013), and that Clowes et al.'s claim on the cosmological implication of existence of the large quasar group needs to be examined quantitatively. Nadathur showed that the algorithm used to identify the quasar groups frequently finds such large-size structures even in homogeneous simulations of a Poisson point process with the same number density as the quasar catalog. They concluded that Clowes et al.'s interpretation of U1.27 as a physical 'structure' is misleading.

Even though it is cosmologically interesting to search for associations of quasars ${ }^{2}$ (Webster 1982; Clowes \& Campusano 1991; Komberg et al. 1996; Williger et al. 2002; Clowes et al. 2012, 2013; Einasto et al. 2014), one needs to be careful in interpreting the quasar groups identified with specific criteria. This is because the size and richness of LSS depend sensitively on how they are identified. Evolution of the objects used to trace LSS and evolution of LSS themselves can also complicate the interpretation of identified LSS. In particular, the quasar phenomenon has short lifetime and astrophysics of quasar activity can affect the results (Komberg et al. 1996; Wold et al. 2000; Miller et al. 2004; Sochting et al. 2004; Coldwell \& Lambas 2006; Hutchings et al. 2009; Lietzen et al. 2009, 2011; Bradshaw et al. 2011; Portinari et al. 2012; Trainor \& Steidel 2012; krumpe et al. 2013; Fanidakis et al. 2013; Shen et al. 2013; DiPompeo et al. 2014; Karhunen et al. 2014; Song et al. 2015). Quasar samples usually have very low spatial number density, which can introduce shot noise effects in group identification. This situation demands the use of welldefined samples and rigorous statistical tests.

In this paper we identify quasar groups using a volume-limited sample drawn from the SDSS DR7 quasar catalog that has the maximum contiguous angular size. We present the statistical significance of not only the largest quasar group, but also the quasar groups within a large range of richness. We also address a question of whether or not the huge quasar group found by Clowes et al. is a statistically-significant object in terms of multiplicity and size. We find the critical linking length (LL hereafter) for quasar group identification which can result in a catalog dominated by physically clustered groups.

\section{SDSS Quasar Catalog}

We use the fifth edition of the SDSS quasar catalog from SDSS DR7, which is a compilation of quasars probed in the SDSS-I and SDSS-II quasar survey (Schneider et al. 2010). The catalog contains 105783 spectroscopically

\footnotetext{
${ }^{2}$ In this paper we call the large loose groupings of quasars 'quasar groups.' This terminology can give a misleading idea that they are compact and physically interacting internally as in galaxy groups. Einasto et al. (2014) used 'quasar systems' to avoid the confusion. The name 'quasar association' may also be an appropriate name for these loose quasar groups since the name 'stellar association' has been already used in astronomy for loose groups of stars that are not gravitationally bound.
}

confirmed quasars in the sky of area covering approximately $9380 \mathrm{deg}^{2}$. They are brighter than $M_{i}=-22.0$ (corrected for the Galactic extinction, and $K$-corrected to $z=2$ in a cosmology with $H_{0}=70 \mathrm{~km} / \mathrm{s} / \mathrm{Mpc}$, $\Omega_{m}=0.3$, and $\Omega_{\Lambda}=0.7$ ) and have at least one broad emission line with full width at half maximum larger than $1000 \mathrm{~km} / \mathrm{s}$ or interesting/complex absorption features. The quasars show a wide redshift distribution from 0.065 to 5.46 , but the majority of quasars $(76 \%)$ is concentrated below redshift 2 . They have the apparent $i$ magnitude of $14.86<i<22.36$, where the lower limit comes from the maximum brightness limit of the target selection on quasar candidates to avoid saturation and cross-talk in the spectra. The catalog does not include several classes of active galactic nuclei such as Type 2 quasars, Seyfert galaxies, and BL Lacertae objects.

Even though the catalog contains a large number of quasars, not all the quasars are suitable for statistical analyses for several reasons. It is mainly because the quasar target selection for the follow-up spectroscopy was not done uniformly in its early version (see Richards et al. 2006; Shen et al. 2007, for more details). Shen et al. (2011) provided a parameter, in the 10 th column in their catalog, named as 'uniform flag' to take the uniformity problem into account. The catalog of Schneider et al. also has its own uniform flag in the 35 th column. The uniform flag is assigned based on the achievement of the target selection algorithm. But Shen et al. made more careful evaluation of the selection effects by finding out quasars that are uniformly selected as high- $z$ candidates but later identified as low$z$ quasars. Shen et al. has one more class than Schneider et al. as follows: $0=$ not in the uniform sample; $1=$ uniformly selected and with the galactic extinctioncorrected $i$ magnitude less than 19.1 at $z<2.9$ or 20.2 at $z>2.9 ; 2=$ uniformly selected by the QSO_HiZ branch in the algorithm and with measured spectroscopic redshift $z<2.9$ and $i>19.1$. By selecting those whose Shen et al. (2011)'s uniform flag is 1, we construct a uniform sample of 59679 quasars with Galactic extinction corrected $i$ magnitude less than 19.1. It rejects about a half of the quasars in the original catalog, mostly at $z<2.9$.

In Figure 1 we plot the quasars in the Schneider et al. (2010)'s catalog in black. Only the northern hemisphere of the SDSS survey regions is adopted and the SDSS survey coordinates $\eta$ and $\lambda$ are used for the plot as the survey boundaries are more simply defined in this coordinate system. The red points in the figure are quasars marked as those of the uniform subsample by Shen et al.

Since the sky distribution of the uniform quasar sample is not perfectly contiguous, we define a new boundary for our sample by excluding isolated patches and trimming the jagged boundaries to make one clean contiguous region. The green line in Figure 1 defines the region under our study. The area of the region is 1.754 steradian. This is nearly the maximum possible contiguous sample that can be drawn from the DR7 cata- 


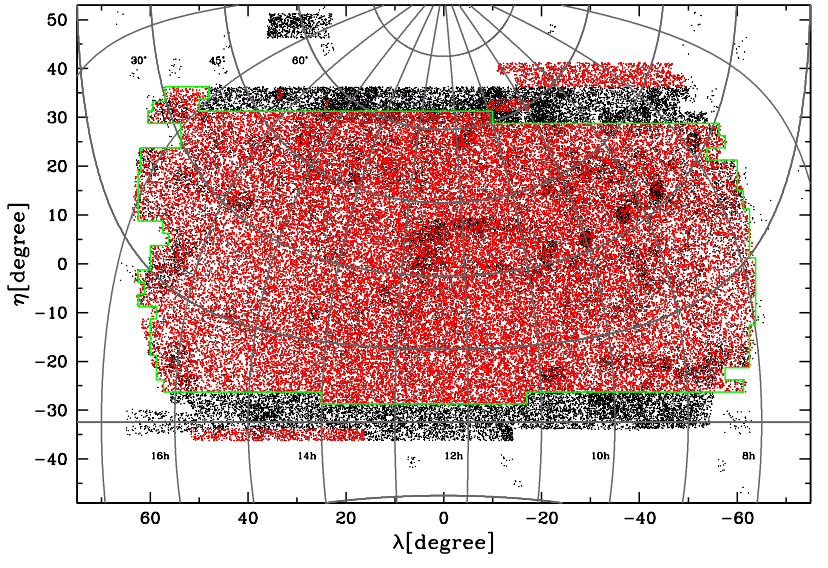

Figure 1. Distribution of all the quasars in the original Schneider et al. (2010)'s catalog (black or red points) and the quasars in the Shen et al. (2011)'s uniform sample (red points) in the SDSS survey coordinates. A contiguous region defined by the green line is the region under analysis in our study.

$\log$.

Figure 2 shows the uniform-sample quasars located within our mask in the $i$-band absolute magnitude and redshift plane. In Figure 3 it can be seen that the comoving space number density of quasars is roughly uniform between $z=0.8$ and 1.8 , but is significantly higher between $z=0.1$ and 0.8 .

Song et al. (2015) has compared the distribution of the SDSS quasars at $0.4 \leq z \leq 0.7$ with the smooth density field of the galaxies in the SDSS DR12 BOSS survey and found that the probability of finding a quasar increases monotonically as the local galaxy number density increases with no significant redshift dependence. This implies that LSS found in the distribution of quasars should be similar to those in the galaxy distribution. Then a quasar sample having a constant comoving number density should be as useful as a volumelimited galaxy sample in finding LSS.

In order to make a volume-limited quasar sample with uniform density we apply an absolute magnitude cut line given by

$$
M_{i, \lim }=0.7216 z^{2}-3.505 z-22.278
$$

in the range of $0.1<z<0.8$. The cut is shown as a blue line in Figure 2. The resulting subsample has a nearly uniform number density of $1.31 \times 10^{-6}\left(h^{-1} \mathrm{Mpc}\right)^{-3}$ in a wide range of $0.1<z<1.8$ as shown by the red histogram in Figure 3. The corresponding mean quasar separation is $91.5 h^{-1} \mathrm{Mpc}$. By varying the absolute magnitude cut one can effectively correct a quasar sample for the luminosity evolution. The use of uniform comoving density sample makes us sample the same kind of cosmological objects over a wide redshift range.

Our final sample contains 32276 quasars with $i<$ 19.1 in the redshift range $0.1 \leq z \leq 1.8$ covering $5758 \mathrm{deg}^{2}$. On the other hand, Clowes et al. (2013) and Nadathur (2013) did not use the sample made

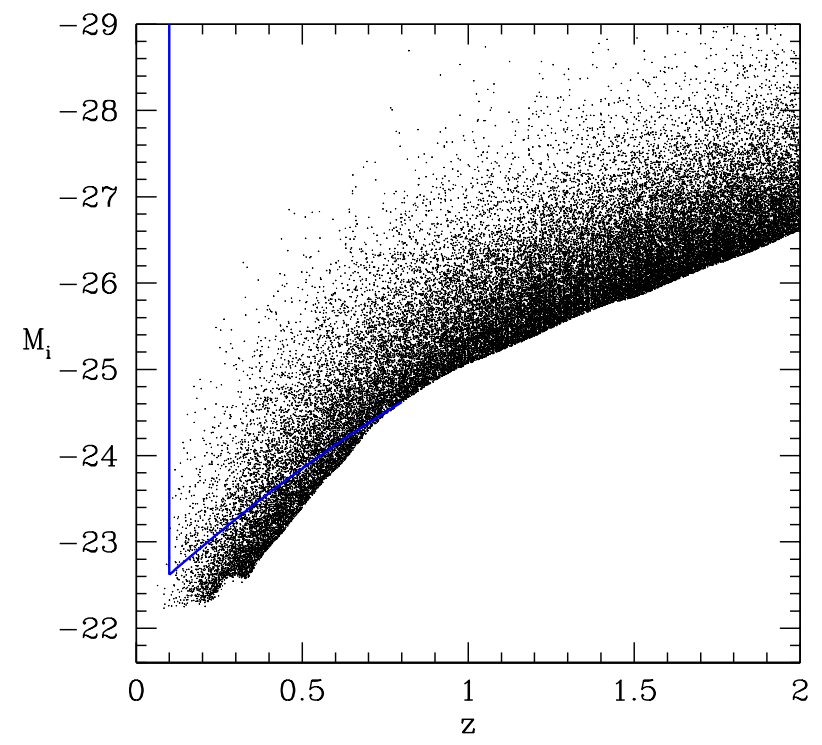

Figure 2. Distribution of the uniform-sample quasars in the redshift versus $i$-band absolute magnitude plane. The blue line is a low-redshift absolute magnitude cut used in our study making the sample nearly constant in comoving number density between $z=0.1$ and 1.8 .

to meet the precondition of uniformity for statistical works. They just applied an $i$-band apparent magnitude cut of $i \leq 19.1$ to Schneider et al. (2010)'s sample, where $i$ here is not corrected for the Galactic extinction. This made their sample affected by non-uniform quasar target selection which Shen et al. (2011) corrected, and the structures identified are affected by the Galactic extinction. Our sample is from the homogeneous sample and has a true flux limit. It is also much larger in the sky area $\left(5758 \mathrm{deg}^{2}\right.$ versus $\left.3725 \mathrm{deg}^{2}\right)$ and in redshift range $(0.1 \leq z \leq 1.8$ versus $1.0 \leq z \leq 1.8)$. It makes our sample contain 1.72 times more quasars than that used by Clowes et al. (2013), Nadathur (2013), and Einasto et al. (2014).

\section{IDENTIFICATION OF QUASAR GROUPS}

To identify groups of quasars we apply the Friendof-Friend (FoF) algorithm to the 'uniform' constantnumber density sample described in the previous section. We first use $\mathrm{LL}=70 h^{-1} \mathrm{Mpc}$ for a comparison with Clowes et al. (2013)'s results. This corresponds to the threshold overdensity of $\delta=(\bar{d} / L L)^{3}-1=$ $(91.5 / 70)^{3}-1=1.23$, where $\bar{d}$ is the mean quasar separation of the sample. We find 3233 quasar groups having 3 or more members. Figure 4 shows the positions of top ten richest groups on the sky (upper panel) and in the $x-y$ plane of Equatorial coordinate frame (bottom panel). The second richest one labelled with ' 2 ' has 70 member quasars and the maximum extent of 703 $h^{-1} \mathrm{Mpc}$. The maximum extent is defined as the comoving distance between the most distant two members in a group. This group corresponds to huge Large Quasar Group, U1.27, reported by Clowes et al. The group 


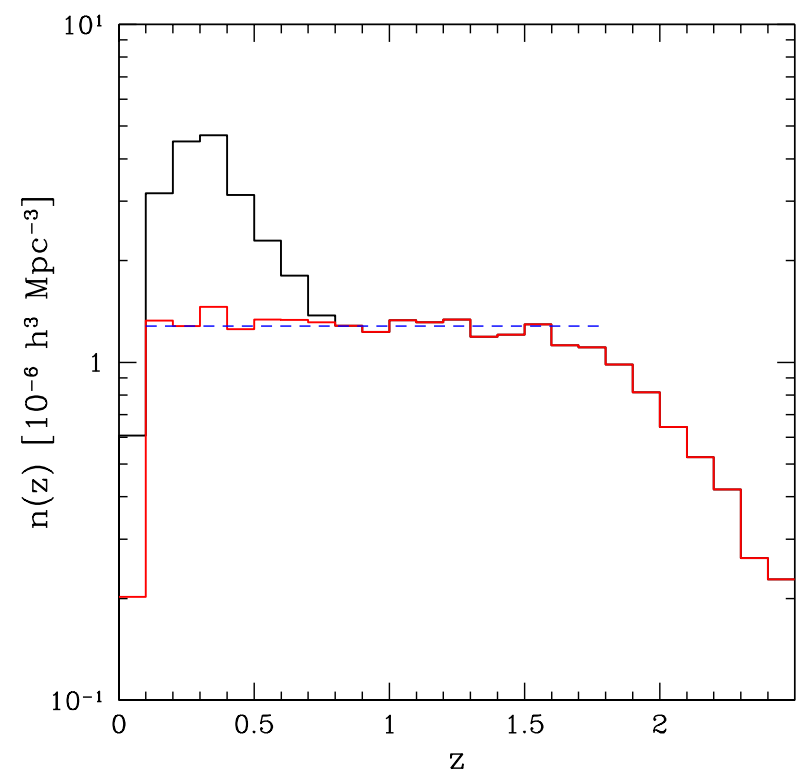

Figure 3. Comoving number density of quasars. The black line is for the whole uniform sample, and the red line is when the low-redshift absolute magnitude cut (Equation (1)) is applied. The blue dashed line is the mean density between $z=0.1$ and 1.8 after the cut is applied.

identified by us has 3 new members and does not have 6 members compared to Clowes et al.'s list. We discover a rich quasar group, marked with ' 1 ' in Figure 4, that contains 95 members and has the maximum extent of $712 h^{-1} \mathrm{Mpc}$. This group was not found by previous studies because its center is located at $z=0.366$, which is outside the redshift limit of $1.0<z<1.8$ adopted by Clowes et al., Nadathur (2013), and Einasto et al. (2014). This group is much richer and has a size larger than U1.27. A catalog of the twenty richest quasar groups is given in Table 1 for future analyses.

To check if our group finding is consistent with previous works we perform the FoF group finding to a sample from the original Schneider et al. (2010)'s catalog that is limited only by $i<19.1$ not corrected for Galactic extinction and a redshift range of $1.0<z<1.8$. In this case the richest group is found to have 73 members that are exactly the same as the member quasars of U1.27 listed in Table 1 of Clowes et al. This proves that the difference in our quasar catalog is not due to the group finding algorithm but to the sample definition. We also compared our quasar groups with those identified by Einasto et al. (2014, see their Table A.3) who also used Schneider et al.'s catalog constrained by $i<19.1$, and found a very good agreement at $z>1.0$. We choose our sample instead of that used by previous works because the observed sample must be uniformly defined for statistical hypothesis tests against random catalogs.

The observed quasar distribution has random perturbations along the line-of-sight due to the random redshift error which is typically about 0.004 in the case of SDSS DR7 catalog (Schneider et al. 2010). Therefore,
Table 1

The twenty richest SDSS DR7 quasar groups identified with the linking length of $70 h^{-1} \mathrm{Mpc}$ or overdensity threshold of 1.23 .

\begin{tabular}{ccccccc}
\hline ID & $\bar{\alpha}(\operatorname{deg})^{\mathrm{a}}$ & $\bar{\delta}(\mathrm{deg})^{\mathrm{a}}$ & $\bar{z}^{\mathrm{a}}$ & $\bar{r}^{\mathrm{a}}$ & $\mathrm{N}^{\mathrm{b}}$ & $\mathrm{L}^{\mathrm{c}}$ \\
\hline 1 & 170.08 & 29.33 & 0.366 & 1012 & 95 & 712 \\
2 & 163.53 & 14.47 & 1.244 & 2757 & 70 & 703 \\
3 & 196.45 & 39.95 & 1.112 & 2546 & 64 & 536 \\
4 & 195.98 & 27.09 & 1.554 & 3198 & 62 & 625 \\
5 & 166.91 & 33.88 & 1.075 & 2485 & 60 & 570 \\
6 & 156.69 & 38.63 & 0.580 & 1519 & 59 & 536 \\
7 & 219.41 & 27.68 & 0.598 & 1560 & 56 & 459 \\
8 & 204.40 & 13.04 & 1.215 & 2712 & 55 & 582 \\
9 & 187.60 & 43.55 & 1.404 & 2995 & 53 & 651 \\
10 & 232.82 & 23.19 & 1.511 & 3141 & 53 & 556 \\
11 & 219.69 & 19.68 & 0.764 & 1909 & 53 & 640 \\
12 & 226.57 & 16.83 & 1.062 & 2463 & 51 & 470 \\
13 & 141.58 & 47.90 & 1.157 & 2620 & 50 & 526 \\
14 & 208.63 & 25.81 & 1.236 & 2745 & 48 & 446 \\
15 & 137.82 & 21.70 & 0.957 & 2279 & 47 & 414 \\
16 & 212.06 & 28.23 & 1.080 & 2493 & 47 & 754 \\
17 & 127.45 & 20.13 & 1.351 & 2917 & 46 & 545 \\
18 & 157.40 & 22.01 & 1.493 & 3117 & 45 & 576 \\
19 & 158.17 & 15.82 & 0.744 & 1869 & 45 & 456 \\
20 & 231.00 & 47.84 & 1.508 & 3138 & 44 & 459 \\
\hline
\end{tabular}

The full catalog with information on members of groups can be downloaded at http://astro.kias.re.kr/Quasar/DR7_ 20groups.

a Coordinates of the geometric center of groups. The mean comoving distance $\bar{r}$ is in units of $h^{-1} \mathrm{Mpc}$.

$\mathrm{b}$ The number of member quasars.

c The maximum extent of groups in $h^{-1} \mathrm{Mpc}$.

it is necessary to examine if the quasar group finding is significantly affected by this error. We will discuss it in the next section.

\section{CoMparison With RANDOM Distributions}

We generate random point sets which have the same number density and the same angular coverage on the sky as our quasar sample. The random catalogs also have the mean point separation of $91.5 h^{-1} \mathrm{Mpc}$ throughout the entire redshift range from $z=0.1$ to 1.8 . We make 10000 random mock samples. For each mock sample we find groups of points in the same way as for the quasar sample. We then measure richness and size of the groups in quasar sample and random mock samples. Richness is the number of member quasars/points belonging to a given group. Size is the maximum comoving extent of the group.

In the upper panel of Figure 5 we plot the cumulative distribution function (CDF) of richness. It is the number of groups whose richness is greater than or equal to $N_{g}$. The circles are from the observed quasar sample. Richness of the group corresponding to U1.27 group is 70 , and is marked with an arrow. The solid line and error bars are the mean and standard deviations obtained from 10000 random catalogs. Similarly, the bottom panel shows the number of observed quasar groups whose size is greater than or equal to L, and the results from 10000 mock catalogs. We find that U1.27 is 703 

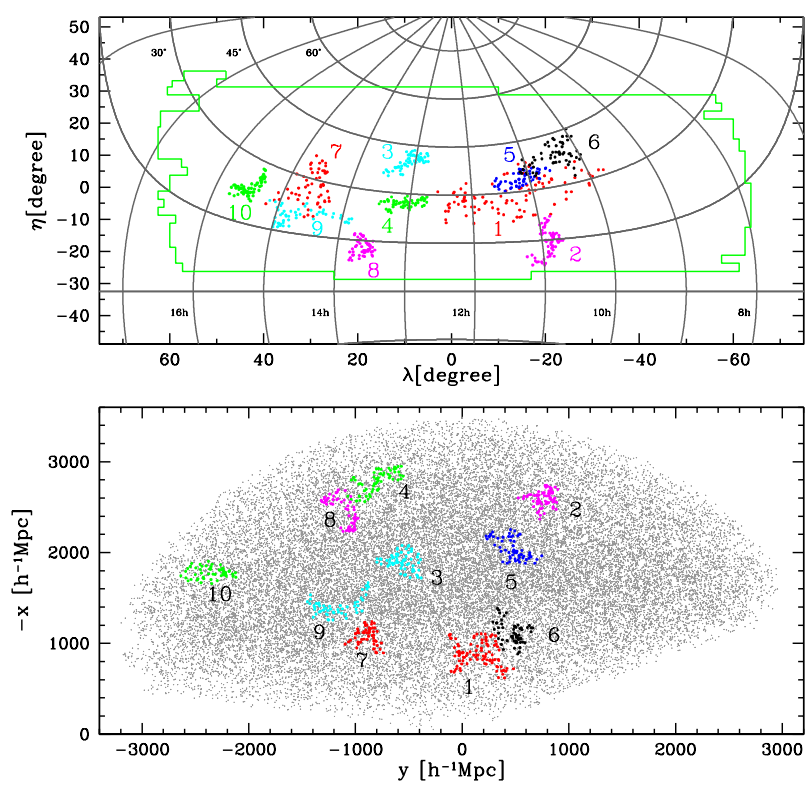

Figure 4. (upper panel) Ten richest quasar groups detected with the linking length of $70 h^{-1} \mathrm{Mpc}$. The second richest one in magenta on the bottom right labelled with 2 corresponds to the huge Large Quasar Group, U1.27, of Clowes et al. (2013). (bottom panel) Same as the upper plot but in the Cartesian coordinates defined in the Equatorial coordinate system. Grey points are all the quasars in our sample.

$h^{-1} \mathrm{Mpc}$ long (marked with an arrow) and is only the fourth largest one. It can be seen that the richness and size distributions of the observed quasars are statistically indistinguishable with those of random catalogs.

To estimate the probability of finding groups like the observed quasar groups in random catalogs we perform a $\chi^{2}$ test. We define

$$
\chi^{2}=\frac{1}{N} \sum_{i=1}^{N}\left(\phi_{i}-\bar{\phi}_{i}\right)^{2} / \sigma_{i}^{2}
$$

as a measure of the deviation of a particular CDF $\phi$ from the average CDF $\bar{\phi}$ of random catalogs. Here $\sigma_{i}$ is the standard deviation of the CDFs of random catalogs in the $i$-th bin, and the summation is over the range where the average CDF of random catalogs is between 3 and 100. The choice ensures that a large part of CDF is compared. The histograms in Figure 6 are the distributions of $\chi^{2}$ of 10000 random catalogs and the arrows correspond to the observed SDSS quasar catalog generated with $\mathrm{LL}=70 \mathrm{~h}^{-1} \mathrm{Mpc}$. The probability of having $\chi^{2}$ greater than $\chi_{\text {obs }}^{2}$ in random catalogs is $58.1 \%$ and $92.4 \%$ for richness and size distributions, respectively. Therefore, it can be concluded that the quasar groups identified with $\mathrm{LL}=70 h^{-1} \mathrm{Mpc}$ are consistent with those of random point distributions.

On the other hand, we find that the probability of finding a random group whose richness is equal to or greater than 70 is $92.3 \%$. Similarly, the probability of finding a random group with the maximum extent equal to or greater than $703 h^{-1} \mathrm{Mpc}$ is $100 \%$. Therefore,
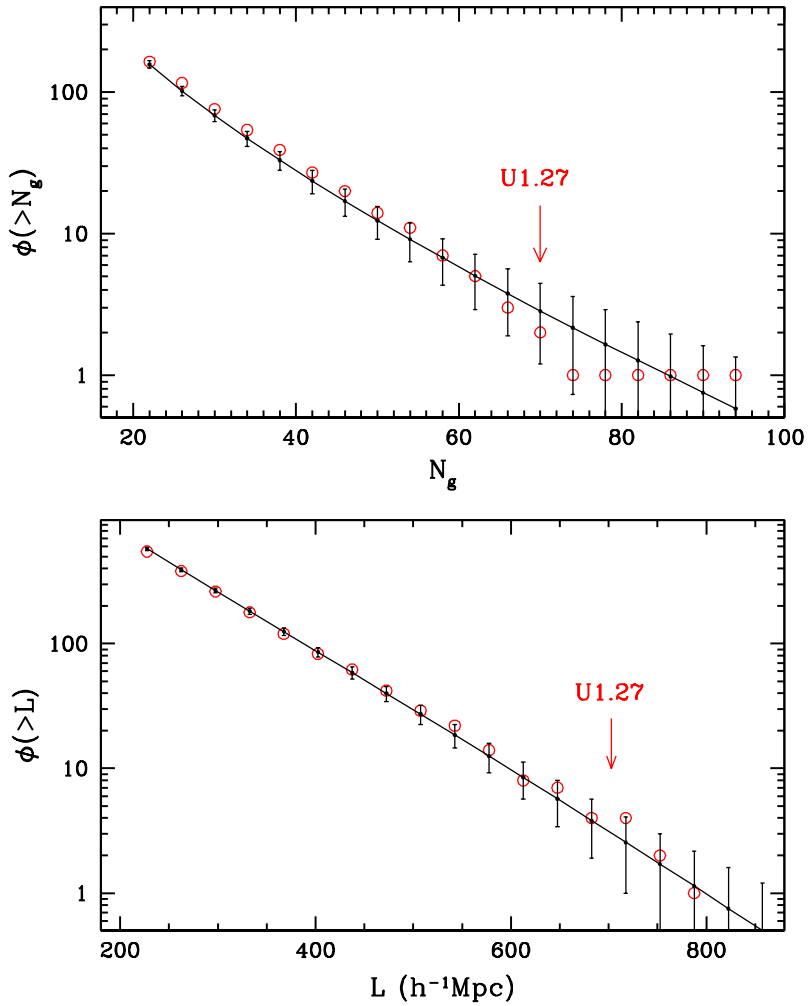

Figure 5. Cumulative distribution functions of richness (upper panel) and maximum extent (bottom panel) of the groups identified in the observed SDSS quasar catalog (red circles) and in random point catalogs (solid line with error bars). A set of 10000 random point catalogs is used for the comparison. The group corresponding to the huge Large Quasar Group, U1.27, reported by Clowes et al. (2013) is marked with downward arrows.

both richness and size of the objects like U1.27 are of no surprise even in random distributions of points with no physical clustering when LL is as large as $70 h^{-1} \mathrm{Mpc}$.

Since quasars do have intrinsic spatial clustering, it is expected that these distribution functions are statistically different from those of random catalogs for some smaller LL or for higher density thresholds. Figure 7 shows the CDFs of richness and size of quasar groups when LL is taken to be 60,50 , and $40 h^{-1} \mathrm{Mpc}$. Also shown are the average CDFs (solid lines with error bars) of random point groups identified with the corresponding LL. It is clear that the CDFs of observed group richness and size become more different from those of random catalogs as LL decreases.

It will be then interesting to search for the critical LL for which the observed CDF becomes significantly different from those of random points. We calculate the probability that the CDF of random point groups happens to show a deviation from the mean CDF as large as or greater than the difference of the observed CDF from the mean. This is estimated by counting the cases with $\chi_{\text {ran }}^{2} \geq \chi_{\text {obs }}^{2}$. A high probability means that the observed groups are statistically consistent with random ones. Figure 8 shows the probability as a function of LL 

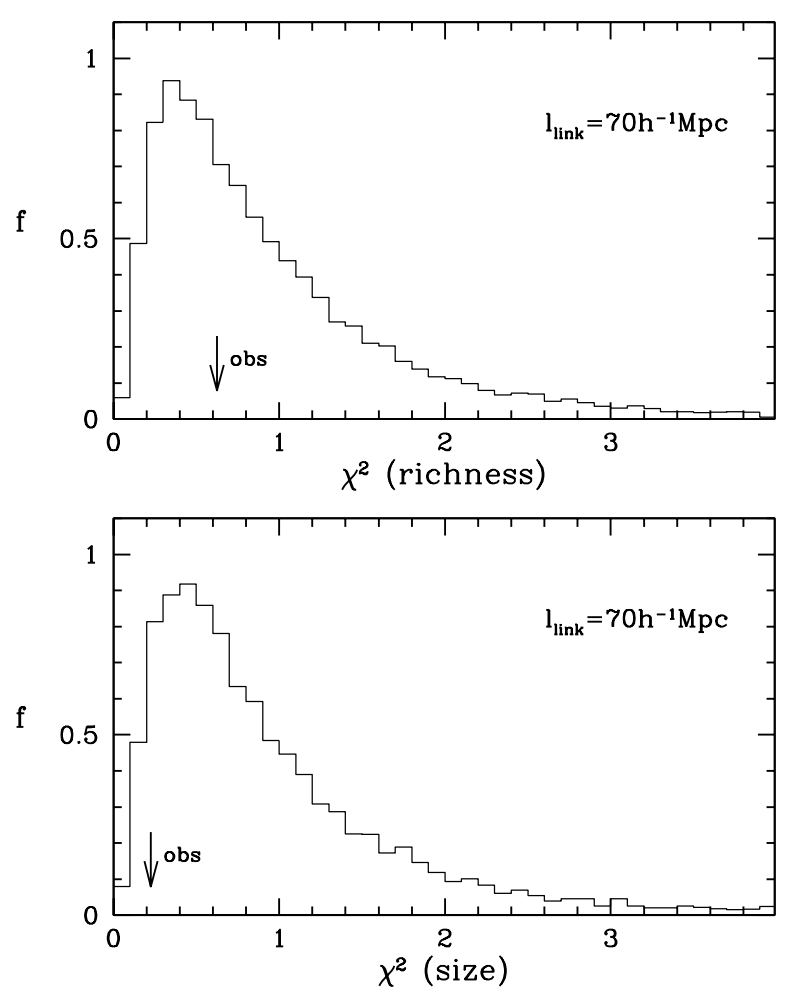

Figure 6. Distributions of $\chi^{2}$ defined as in Equation (2) for random point samples. The upper panel is for richness of groups identified with the linking length of $70 h^{-1} \mathrm{Mpc}$, and the bottom panel is for maximum extent of groups. The $\chi^{2}$ for the observed sample are marked with arrows.

used to find groups. For both richness and size it can be seen that the observed groups are consistent with those of random catalogs when LL is chosen to be larger than about $50 h^{-1} \mathrm{Mpc}$. Clowes et al. chose $\mathrm{LL}=70 h^{-1} \mathrm{Mpc}$ and it is now clear that the choice makes the group catalog dominated by randomly connected groups. Figures 7 and 8 indicate that the groups identified with $\mathrm{LL}<50 h^{-1} \mathrm{Mpc}$ are likely to be physically-clustered genuine groups of quasars.

As mentioned in the previous section the random redshift error of order of 0.004 seems problematic in finding physical structures as the error corresponds to $\sim$ $12 h^{-1} \mathrm{Mpc}$. We use the redshifts calculated by Hewett \& Wild (2010, hereafter HW10) whose redshift errors are much smaller and redo the group findings. According to the error estimates for the redshifts of HW10, the RMS redshift error for quasars at $0.1<z<1.8$ is found to be only 0.00024 , more than 10 times smaller than the nominal value for the SDSS DR7.

It will be a good consistency check to compare two group catalogs derived from the redshifts from Schneider et al. (2010) and HW10. We find that the new quasar group catalog is very close to the previous one obtained using Schneider et al. (2010)'s redshifts. All major groups are detected again and only a few members are affected. As a result, the richness and size distributions change only slightly. It is reassuring that the quasar groups and the statistics are robust against
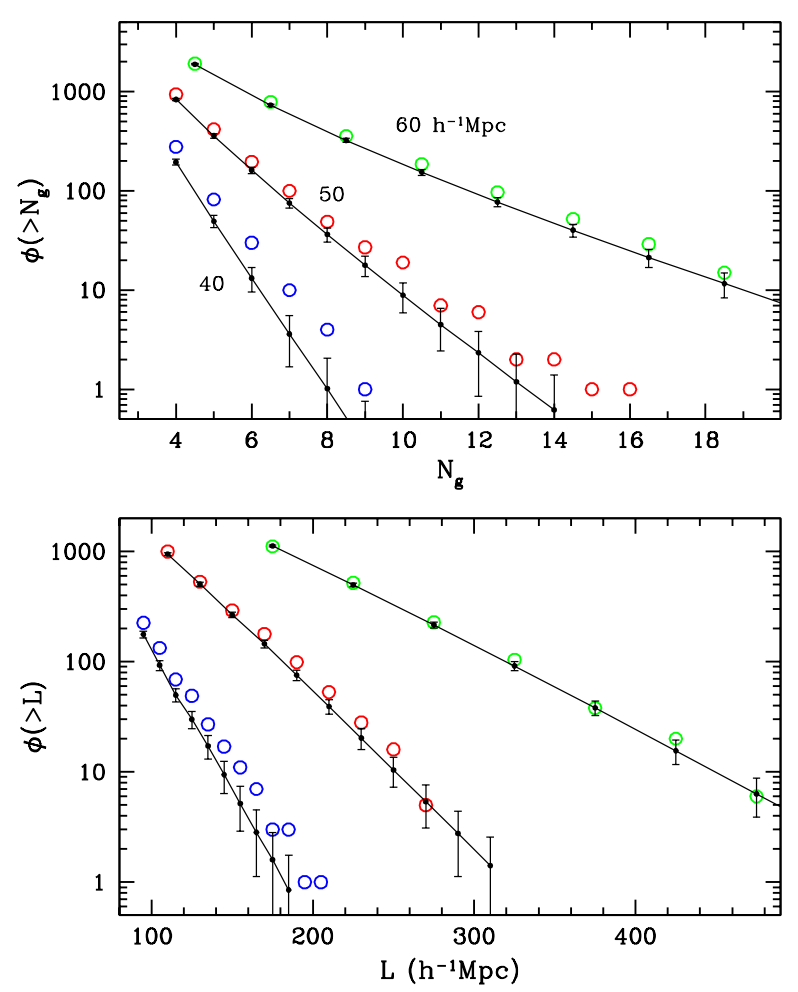

Figure 7. Cumulative distribution functions of richness (upper panel) and maximum extent (bottom panel) when the linking length for group identification is taken to be 60,50 , and $40 h^{-1} \mathrm{Mpc}$. Circles are from observation, and solid lines with error bars are from 1000 random catalogs.

the redshift error of Schneider et al. (2010). When $L L=50 h^{-1} \mathrm{Mpc}$, we still find a very good agreement of both distribution functions. Therefore, all of our results are essentially unchanged by using more accurate quasar redshifts. We stick to Schneider et al. (2010)'s redshifts in our paper since there is practically no change in the conclusions and it is easier to compare our groups directly to those of previous works. The groups identified in the redshift space are also affected by the peculiar velocities of quasars. Influence of peculiar velocity on group identification is to some extent unavoidable. But this is relatively small because the RMS pairwise peculiar velocity of galaxies is only about $300 \mathrm{~km} / \mathrm{s}$.

\section{Summary AND CONCluSions}

We make a wide contiguous volume-limited sample of quasars statistically uniformly selected from the SDSS DR7 quasar catalog and identify quasar groups using the FoF algorithm. The statistical properties of the observed quasar groups are compared with those of random point sets. We make the following findings.

1. The observed SDSS DR7 quasar groups identified with $\mathrm{LL}=70 \mathrm{~h}^{-1} \mathrm{Mpc}$ are statistically consistent, in terms of size and richness, with those of random points clustered by chance. The size or richness distribution functions of those quasar groups can be found in ran- 


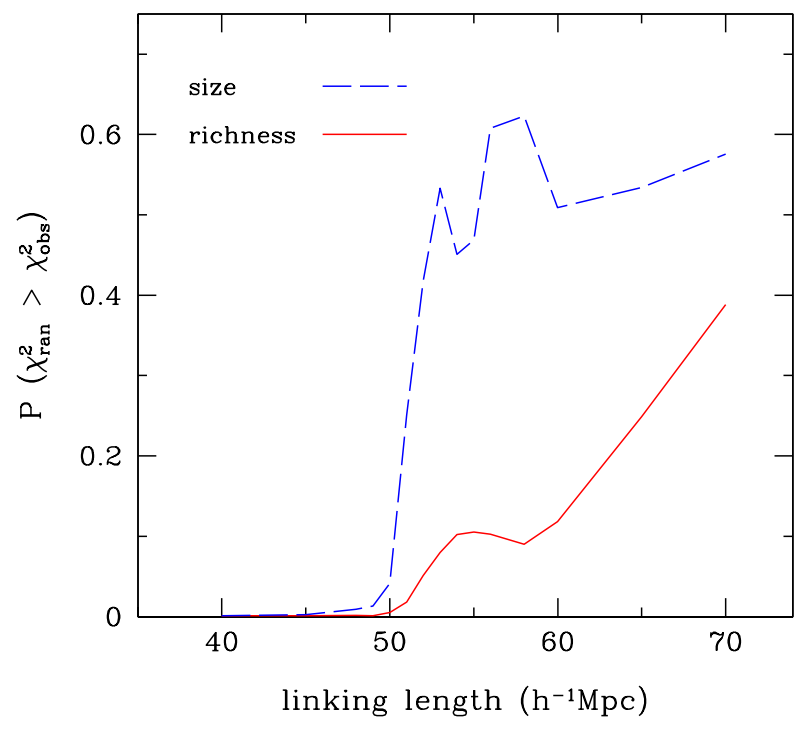

Figure 8. Probability of finding random catalogs whose distribution function deviates from their mean more than or equal to that of the observed sample. The deviation is measured by $\chi^{2}$ defined in Equation (2). Probability is measured for richness (solid line) and size (dashed line) distribution functions as a function linking length.

dom catalogs with $92.4 \%$ and $58.1 \%$ probabilities, respectively.

2. The probabilities of finding groups larger or richer than the large quasar group U1.27, reported by Clowes et al. (2013), in a random point catalog are $100 \%$ and $92 \%$, respectively.

3. The quasar groups are statistically dominated by physically clustered quasars when LL for group identification is less than $50 h^{-1} \mathrm{Mpc}$.

We emphasize that the observed LSS should not be used to draw cosmological conclusions without making elaborate statistical tests. Size and richness of cosmic structures depend sensitively on how those structures are identified. For example, one can always find structures as large as the survey size if the threshold density level used to identify them is lowered close to the percolation density. Even in a random point distribution without any physical clustering an infinitely long structure can be identified when LL is increased to 0.864 times the mean particle separation, which is the critical value for percolation. It is conceivable that one would claim discovery of groups of galaxies or quasars even larger than the Sloan Great Wall in existing or future redshift surveys. The lesson of the past is that one will again need to make statistical tests before drawing conclusions on cosmological implication of those objects.

Our results suggest that the large quasar groups identified with $\mathrm{LL}>50 \mathrm{~h}^{-1} \mathrm{Mpc}$ can be considered as associations of smaller physically-clustered quasar systems that are connected by a very large linking length. The smaller quasar systems are similar to the supercluster complexes in the local universe, as was suggested in Einasto et al. (2014). Hutsemékers et al. (2014) showed that quasar spin axes in U1.27 seem to be aligned parallel to this structure. However, from their Figure 6 one can see that alignment is better if this quasar group is divided into smaller systems, in agreement with our interpretation of quasar systems.

There have also been several studies trying to define and measure the homogeneity scale of the universe (Yadav et al. 2010; Marinoni et al. 2012; Scrimgeour et al. 2012). However, definition of the homogeneity scale has been either arbitary or dependent on observational samples. We point out that, in the current popular cosmological models that adopt the Cosmological Principle, the notion of the critical scale of homogeneity does not make sense. Homogeneity is only achieved asymptotically in these models as the scale of observation increases, and there is no characteristic scale intrinsic to the universe above which the universe can be suddenly regarded homogeneous (see also Nadathur 2013). Instead of looking for arbitrarily-defined homogeneity scales or naively examining the size of the largest structures the observed LSS should be statistically compared with the structures identified in the same way in the mock survey samples obtained from simulated initiallyhomogeneous universes. In our forthcoming paper we will use mock quasars formed in simulated universes to identify large groups of quasars and statistically compare their properties with those of observed quasars. We will examine if the large structures can be explained in the framework of the standard hierarchical structure formation model in the given Hubble time.

\section{ACKNOWLEDGMents}

The authors thank the anonymous referee for helpful comments and reviewing our paper quickly, and also thank Korea Institute for Advanced Study for providing computing resources (KIAS Center for Advanced Computation Linux Cluster System). M. Einasto was supported by the ETAG project IUT26-2, and by the European Structural Funds grant for the Centre of Excellence "Dark Matter in (Astro)particle Physics and Cosmology" TK120. H. Lietzen acknowledges financial support from the Spanish Ministry of Economy and Competitiveness (MINECO) under the 2011 Severo Ochoa Program MINECO SEV-2011-0187.

Funding for the SDSS and SDSS-II has been provided by the Alfred P. Sloan Foundation, the Participating Institutions, the National Science Foundation, the U.S. Department of Energy, the National Aeronautics and Space Administration, the Japanese Monbukagakusho, the Max Planck Society, and the Higher Education Funding Council for England. The SDSS Web Site is http://www.sdss.org.

\section{REFERENCES}

Bradshaw, E. J., Almaini, O., Hartley, W. G., Chuter, R. W., Simpson, C., Conselice, C. J., Dunlop, J. S., McLure, R. J., \& Cirasuolo, M. 2011, Environments of Active Galactic Nuclei at $z<1.5$ in the UKIDSS Ultra-Deep Survey, MNRAS, 415, 2626 
Clifton, T., Clarkson, C., \& Bull, P. 2012, Isotropic Blackbody Cosmic Microwave Background Radiation as Evidence for a Homogeneous Universe, PRL, 109, 051303

Clowes, R. G., \& Campusano, L. E. 1991, A 100-200 MPC Group of Quasars, MNRAS, 249, 218

Clowes, R. G., Campusano, L. E., Graham, M. J., \& Sochting, I. K. 2012, Two Close Large Quasar Groups of Size $\sim 350 \mathrm{Mpc}$ at $z \sim 1.2$, MNRAS, 419, 556

Clowes, R. G., Harris, K. A., Raghunathan, S., et al. 2013, A Structure in the Early Universe at $z \sim 1.3$ that Exceeds the Homogeneity Scale of the R-W Concordance Cosmology, MNRAS, 429, 2910

Coldwell, G. V., \& Lambas, D. G. 2006, Properties of Galaxies in Sloan Digital Sky Survey Quasar Environments at $z<0.2$, MNRAS, 371, 786

de Lapparent, V., Geller, M. J., \& Huchra, J. P. 1986, A Slice of the Universe, ApJ, 302, L1

Einasto, M., Liivamägi, L. J., Tempel, E., Saar, E., Tago, E., Einasto, P., Enkvist, I., Einasto, J., Martínez, V. J., Heinämäki, P., \& Nurmi, P. 2011, The Sloan Great Wall. Morphology and Galaxy Content, ApJ, 736, 51

Einasto, M., Tago, E., Lietzen, H., Park, C., Heinämäki, P., Saar, E., Song, H., Liivamägi, L. J., \& Einasto, J. 2014, Tracing a High Redshift Cosmic Web with Quasar Systems, A\&A, 568, 46

Geller, M. J., \& Huchra, J. P. 1989, Mapping the Universe, Science, 246, 897

DiPompeo, M. A., Myers, A. D., Hickox, R. C., Geach, J. E., \& Hainline, K. N. 2014, The Angular Clustering of Infrared-Selected Obscured and Unobscured Quasars, MNRAS, 442, 3443

Fanidakis, N., Maccio, A. V., Baugh, C. M., Lacey, C. G., \& Frenk, C. S. 2013, The Most Luminous Quasars Do Not Live in the Most Massive Dark Matter Haloes at Any Redshift, MNRAS, 436, 315

Gott, J. R., Jurić, M., Schlegel, D., et al. 2005, A Map of the Universe, ApJ, 624, 463

Hewett, P. C., \& Wild, V. 2010, Improved Redshifts for SDSS Quasar Spectra, MNRAS, 405, 2302

Horvath, I., Hakkila, J., \& Bagoly, Z. 2013, The Largest Structure of the Universe, Defined by Gamma-Ray Bursts, arXiv1311.1104

Hutchings, J. B., Scholz, P., \& Bianchi, L. 2009, Environments of QSOs at Redshift 0.9-1.3, AJ, 137, 3533

Hutsemékers, D., Braibant, L., Pelgrims, V., \& Sluse, D. 2014, Alignment of Quasar Polarizations with Large-Scale Structures, A\&A, 572, 18

Karhunen, K., Kotilainen, J. K., Falomo, R., \& Bettoni, D. 2014, Low-Redshift Quasars in the SDSS Stripe 82. The Local Environments, MNRAS, 441, 1802

Komberg, B. V., Kravtsov, A. V., \& Lukash, V. N. 1996, The Search for and Investigation of Large Quasar Groups, MNRAS, 282, 713

Krumpe, M., Miyaji, T., \& Coil, A. L. 2013, Clustering Measurements of broad-Line AGNs: Review and Future, arXiv1308.5976

Lietzen, H., Heinämäki, P., Nurmi, P., Liivamagi, L. J., Saar, E., Tago, E., Takalo, L. O., \& Einasto, M. 2011, Large-Scale Environments of $z<0.4$ Active Galaxies, A\&A, 535, 21

Lietzen, H., Heinämäki, P., Nurmi, P., Tago, E., Saar, E., Liivamagi, J., Tempel, E., Einasto, M., Einasto, J., Gramann, M., \& Takalo, L. O. 2009, Environments of Nearby
Quasars in Sloan Digital Sky Survey, A\&A, 501, 145

Marinoni, C., Bel, J., \& Buzzi, A. 2012, The Scale of Cosmic Isotropy, JCAP, 10, 036

Miller, L., Croom, S. M., Boyle, B. J., Loaring, N. S., Smith, R. J., Shanks, T., \& Outram, P. 2004, 200-Mpc-Sized Structure in the 2dF QSO Redshift Survey, MNRAS, 355, 385

Nadathur, S. 2013, Seeing Patterns in Noise: GigaparsecScale 'Structures' that Do Not Violate Homogeneity, MNRAS, 434, 398

Park, C. 1990, Large N-Body Simulations of a Universe Dominated by Cold Dark Matter, MNRAS, 242, 59P

Park, C., Choi, Y.-Y., Kim, J., Gott, J. R., Kim, S. S., \& Kim, K.-S. 2012, The Challenge of the Largest Structures in the Universe to Cosmology, ApJ, 759, L7

Planck Collaboration, Ade, P. A. R., Aghanim, N., et al. 2014, Planck 2013 Results. XV. CMB Power Spectra and Likelihood, A\&A, 571, 15

Portinari, L., Kotilainen, J., Falomo, R., \& Decarli, R. 2012, On the Cosmological Evolution of the Black Hole-Host Galaxy Relation in Quasars, MNRAS, 420, 732

Richards, G. T., Strauss, M. A., Fan, X., et al. 2006, The Sloan Digital Sky Survey Quasar Survey: Quasar Luminosity Function from Data Release 3, AJ, 131, 2766

Schneider, D. P., Richards, G. T., Hall, P. B., et al. 2010, The Sloan Digital Sky Survey Quasar Catalog. V. Seventh Data Release, AJ, 139, 2360

Scrimgeour, M. I., Davis, T., Blake, C., et al. 2012, The WiggleZ Dark Energy Survey: the Transition to LargeScale Cosmic Homogeneity, MNRAS, 425, 116

Shen, Y., Richards, G. T., Strauss, M. A., et al. 2011, A Catalog of Quasar Properties from Sloan Digital Sky Survey Data Release 7, ApJS, 194, 45

Shen, Y., McBride, C. K., White, M., et al. 2013, CrossCorrelation of SDSS DR7 Quasars and DR10 BOSS Galaxies: The Weak Luminosity Dependence of Quasar Clustering at $z \sim 0.5$, ApJ, 778, 98

Shen, Y., Strauss, M. A., Oguri, M., et al. 2007, Clustering of High-Redshift $(z \geq 2.9)$ Quasars from the Sloan Digital Sky Survey, AJ, 133, 2222

Sheth, R. K., \& Diaferio, A. 2011, How Unusual Are the Shapley Supercluster and the Sloan Great Wall?, MNRAS, 417, 2938

Sochting, I. K., Clowes, R. G., \& Campusano, L. E. 2004, Relation of Radio-Quiet Quasars to Galaxy Clusters at $z<0.3$, MNRAS, 347, 1241

Song, H., Park, C., Lietzen, H., \& Einasto, M. 2015, in preparation

Trainor, R. F., \& Steidel, C. C. 2012, The Halo Masses and Galaxy Environments of Hyperluminous QSOs at $z \sim 2.7$ in the Keck Baryonic Structure Survey, ApJ, 752, 39

Webster, A. 1982, The Clustering of Quasars from an Objective-Prism Survey, MNRAS, 199, 683

Williger, G. M., Campusano, L. E., Clowes, R. G., \& Graham, M. J. 2002, Large-Scale Structure at $z=1.2$ Outlined by Mg II Absorbers, ApJ, 578, 708

Wold, M., Lacy, M., Lilje, P. B., \& Serjeant, S. 2000, Clustering of Galaxies around Radio Quasars at $0.5 \leq z \leq 0.8$, MNRAS, 316, 267

Yadav, J. K., Bagla, J. S., \& Khandai, N. 2010, Fractal Dimension as a Measure of the Scale of Homogeneity, MNRAS, 405, 2009 